\title{
Numerical study of pattern formation following a convective instability in non-Boussinesq fluids
}

\author{
Hao-wen Xi \\ Department of Physics \\ Lehigh University \\ Bethlehem, Pennsylvania 18015, \\ Jorge Viñals \\ Supercomputer Computations Research Institute, B-186 \\ and \\ Department of Chemical Engineering, B-203 \\ Florida State University \\ Tallahassee, Florida 32306-4052, \\ and \\ J.D. Gunton \\ Department of Physics \\ Lehigh University \\ Bethlehem, Pennsylvania 18015.
}

October 28, 2018 
We present a numerical study of a model of pattern formation following a convective instability in a non-Boussinesq fluid. It is shown that many of the features observed in convection experiments conducted on $\mathrm{CO}_{2}$ gas can be reproduced by using a generalized two-dimensional Swift-Hohenberg equation. The formation of hexagonal patterns, rolls and spirals is studied, as well as the transitions and competition among them. We also study nucleation and growth of hexagonal patterns and find that the front velocity in this two dimensional model is consistent with the prediction of marginal stability theory for one dimensional fronts. 
One of the most natural and intriguing behaviors of complex systems driven far from thermal equilibrium is their ability to undergo symmetry breaking instabilities that lead to the spontaneous formation of spatio-temporal structures. An excellent example is the Rayleigh-Bénard instability. Much of the earlier experimental work has been restricted to Oberbeck-Boussinesq type fluids, in which one observes various configurations of roll patterns. However, in a non-Oberbeck-Boussinesq system with, for example, temperature dependent transport coefficients, both roll and hexagonal patterns can exist. Very recently Bodenschatz et al. [四] have performed experiments on convection in $\mathrm{CO}_{2}$ gas and studied the existence of and transitions between convective patterns exhibiting different symmetries. They have observed the competition between a uniform conducting state, a convective state with hexagonal symmetry, and convecting patterns comprising parallel rolls. In this letter we present the results of a numerical solution of a two dimensional model equation for the case of a large aspect ratio cylindrical cell near onset, and qualitatively compare our results with the experiments of Bodenschatz et al.

Our study is based on a numerical integration of a two dimensional generalized Swift-Hohenberg equation [2],

$$
\frac{\partial \psi(\vec{r}, t)}{\partial t}=\left[\epsilon(\vec{r})-\left(\nabla^{2}+1\right)^{2}-\psi^{2}\right] \psi+g_{2} \psi \nabla^{2} \psi+f(\vec{r})
$$

with boundary conditions,

$$
\left.\psi\right|_{B}=\left.\hat{n} \cdot \nabla \psi\right|_{B}=0
$$

where $\hat{n}$ is the unit normal to the boundary of the domain of integration, $B$. This equation with $g_{2}=0$ reduces to the Swift-Hohenberg equation and has been extensively used to model convection in thin cells and near onset [3, 4, 5, 6]. The scalar order parameter $\mathrm{y}$ is related to the fluid temperature in the mid-plane of the convective cell. The quantity $\epsilon$ is the reduced Rayleigh number,

$$
\epsilon=\frac{R}{R_{c}^{\infty}}-1
$$

where $R$ is the Rayleigh number and $R_{c}^{\infty}$ is the critical Rayleigh number for an infinite 
system. A forcing field $f$ has been added to mimic lateral sidewall forcing (for details, see ref. [7], 8]).

We have derived a three mode amplitude equation from the generalized SH equation in order to both estimate the threshold values of $\epsilon$ that separate regions in which roll and hexagonal configurations are stable, and the values of the parameters that enter the generalized SH equation in terms of experimentally measurable quantities. From the experiments [1], we find that $g_{2} \approx 0.35$. This is the value that we have used throughout our calculations. The value of $\epsilon$ used in the numerical simulation is related to the real experimental value $\epsilon^{\exp }$ in ref. [1] by $\epsilon^{\exp }=0.3594 \epsilon$. The values of $\epsilon$ for which hexagonal and roll patterns are stable can be obtained from the three mode amplitude equation. For our value of $g_{2}$ we find that hexagons are stable for $0<\epsilon<\epsilon_{r}$, with $\epsilon_{r}=0.163$. Hexagonal patterns and rolls coexist for $\epsilon_{r}<\epsilon<\epsilon_{b}$, with $\epsilon_{b}=0.65$. For $\epsilon>\epsilon_{b}$, only rolls are stable. We have studied two different forms for the quandratic term in Eq. (1), $\psi \nabla^{2} \psi$ and $\psi^{2}$. We have found that both models give essentially the same results. In this paper, we report on the results that correspond to $\psi \nabla^{2} \psi$ to study any non-variational effects on the hexagon to roll transition. We have neglected the effect of mean flow [9, 10] and have concentrated on the qualitative features of pattern formation as described by Eq. (1). However, mean flow effects are needed to describe, for example, a rotating spiral pattern, as shown by Bestehorn et al. recently [11]. In the following we report the results of our calculations.

i) Nucleation of a pattern with hexagonal symmetry. We consider as initial condition, $\psi(\vec{r}, t=0)$, a Gaussian random variable with zero mean and variance $10^{-6}$. The forcing field $f(\vec{r})=0$ simply because there is no influence of lateral boundaries before the nucleated pattern reaches the boundary. We numerically solve Eq. (11) in a square domain of side $L=120 \pi$ (in our dimensionless variables, this corresponds to an aspect ratio $\Gamma=L / \pi=120$ ). The differential equation is discretized on a square grid with $512 \times 512$ nodes. We take $\epsilon=0.01$ except in a small square region near the center of the cell (of size $16 \times 16$ nodes) where $\epsilon=0.055$. This space dependent $\epsilon$ models 
a small localized inhomogeneity in one of the cell plates. The temporal evolution of the pattern is shown in Fig. 1a. It presents an early transient behavior during which a local convective region with hexagonal symmetry has just nucleated. Six fronts of rolls are traveling away from the hexagonal patch located at the center. As they propagate further into the conduction region, they spread the convective region with hexagonal symmetry. This is remarkably similar to the experimental observations of Bodenschatz et al. [1]. It is worth pointing out, however, that the shape of the envelope of rolls is triangular in the experiment, whereas it appears to be rectangular in our numerical results. This difference may be attributable to a shortcoming of our model equation. It is also possible, but probably not likely, that we have not used a sufficiently small value of epsilon. We have also estimated the speed of propagation of the front that separates the hexagonal pattern and the uniform state. This speed, at the center of the planar sides, and along their normal direction, is constant in time and equals $v_{\perp}=0.37$. The value given by marginal stability theory for the one dimensional Swift-Hohenberg equation $\left(g_{2}=0\right)$ is $v_{M S}=0.397$ [12, 13]. To our knowledge this front velocity has not yet been experimentally measured. The observation of nucleation and growth is especially interesting since it provides an example of competition among different symmetries, i.e. a uniform conduction state as the background state, a region of hexagonal symmetry being nucleated and rolls in the front region separating the two. This situation is also interesting from the point of view of pattern selection during front propagation in dimensions higher than one.

ii) Formation of patterns with hexagonal symmetry by sidewall forcing. We have considered a circular cell of radius $R=30 \pi$, which corresponds to an aspect ratio of $\Gamma=2 R / \pi=60$. A square grid with $N^{2}$ nodes has been used with spacing $\Delta x=\Delta y=60 \pi / \mathrm{N}$, and $N=256$. We approximate the boundary conditions on $\psi$ by taking $\psi(\vec{r}, t)=0$ for $\|\vec{r}\| \geq R$, where $\vec{r}$ is the location of a node with respect to the center of the domain of integration. The initial condition $\psi(\vec{r}, t=0)$ is a random variable, gaussianly distributed with zero mean and variance $10^{-2}$. In this case $\epsilon=0.1$, and $f=0$ everywhere except on the nodes adjacent to the boundary. 
Two values of $f$ have been studied: $f=0.1$ and $f=0.5$. They will be referred to as weak and strong forcing respectively. Figure 2a presents a typical configuration for the case of weak sidewall forcing. Two ordered domains appear in the cell with different orientation and meet at an abrupt angle, creating the analog of a grain boundary near a diameter of the cell. On the contrary, we find that a hexagonal pattern with concentric orientation is induced spontaneously by the presence of strong sidewall forcing, as shown in Fig. $2 \mathrm{~b}$.

iii) Transition between hexagons and rolls. We use as initial condition the configuration shown in Fig. 2b, with exactly the same forcing field $f$ but take $\epsilon=0.3$. Figure 3 shows a sequence of configurations during the hexagon to roll transition. How defects mediate the transition can also be seen in Fig. 3. They glide towards each other, and invade nearby regions of hexagonal pattern to create a region of rolls that spreads through the cell as the transition proceeds. This is also very close to the experimental observation, except in that Bodenschatz et al. [1] found that there is a tendency towards the bending of rolls and the formation of spirals during the hexagon to roll transition. This tendency does not appear in our numerical results. This may be due to our neglect of mean flow effects in our model.

iv) Stable spiral pattern. A one-armed spiral pattern is shown in Fig. 4. We start with random initial conditions in which $\psi(\vec{r}, t=0)$ is a Gaussian random variable with with zero mean and variance $10^{-8}$. The forcing field at the boundary is $f=0.1$ and $\epsilon=0.2$. The cylindrical cell has an aspect ratio of 60 and we have used $256 \times 256$ grid nodes. We have observed that whenever a defect appears, the neighboring rolls start to buckle and a clear distortion occurs. This deformation develops, ending in a stable spiral pattern. However, we are unable to see any rotation of the spiral, presumably because of the lack of a mean flow term, as stated earlier.

In summary, we have investigated the question of pattern formation in a model of convection in a non-Boussinesq fluid that allows stationary patterns of various 
symmetries. Because the generalized Swift-Hohenberg equation that we have used is related to a non-Boussinesq fluid near the onset of convection, we have been able to investigate several interesting issues relevant to recent experimental observations in $\mathrm{CO}_{2}$ gas. We have studied nucleation of hexagonal patterns, the formation of patterns with hexagonal symmetry, the transition from patterns with hexagonal symmetry to rolls and the formation of a stable spiral pattern. Our numerical calculations have further illustrated the strong influence of sidewall forcing in pattern formation. Approximate boundaries in parameter space separating regions in which patterns of the various symmetries occur, as well as a quantitative study of front propagation will be given in future work.

We wish to thank E. Bodenschatz, G. Ahlers and D. Cannell for suggesting the numerical investigation of the generalized Swift-Hohenberg equation, and them and P. Hohenberg for many stimulating conversations and comments. This work was supported in part by the National Science Foundation under Grant No. DMR-9100245. This work is also supported in part by the Supercomputer Computations Research Institute, which is partially funded by the U.S. Department of Energy contract No. DE-FC05-85ER25000. The calculations reported here have been performed on the 64k-node Connection Machine at the Supercomputer Computations Research Institute and on the Cray Y-MP at the Pittsburgh Supercomputing Center.

\section{References}

[1] E. Bodenschatz, J.R. de Bruyn, G. Ahlers and D.S. Cannell, Phys. Rev. Lett. 67, 3078 (1991).

[2] J. Swift and P.C. Hohenberg, Phys. Rev. A 15, 319 (1977).

[3] P. Collet and J.-P. Eckman, Instabilities and Fronts in Extended Systems, (Princeton University Press, New Jersey, 1990). 
[4] P. Manneville, Dissipative Structures and Weak Turbulence, (Academic, New York, 1990).

[5] M.C. Cross, Phys. Fluids 23, 1727 (1980); Phys. Rev. A 25, 1065 (1982); Phys. Rev. A 27, 490 (1983).

[6] H.S. Greenside and W.M. Coughran Jr., 49, 726 (1982); H.S. Greenside and W.M. Coughran Jr., Phys. Rev. A 30, 398 (1984); H.S. Greenside and M.C. Cross, Phys. Rev. A 31, 2492 (1985).

[7] H.W. Xi, J. Viñals and J.D. Gunton, Physica A 177, 356 (1991).

[8] J. Viñals, H.W. Xi and J.D. Gunton, Phys. Rev. A, in press.

[9] E.D. Siggia and A. Zippelius, Phys. Rev. Lett. 47, 835 (1981); A. Zippelius and E.D. Siggia, Phys. Rev, A 26, 178 (1982).

[10] P. Manneville, J. Physique 44, 759 (1983).

[11] M. Bestehorn, M. Fantz, R. Friedrich, H. Haken and C. Pérez-García, unpublished.

[12] G. Dee and J.S. Langer, Phys. Rev. Lett. 50, 383 (1983).

[13] W. van Saarloos, Phys. Rev. A 37, 211 (1988). 


\section{Figure captions}

Figure 1. Nucleation of a pattern with hexagonal symmetry in a square cell with aspect ratio $\Gamma=120$. The values of the parameters used are $g_{2}=0.35, \epsilon=0.01$ and $f=0$. In a small square region at the center of the cell, $\epsilon=0.055$. The time shown is $t=611$ and dark (white) areas represent regions in which $\psi$ is positive (negative).

Figure 2. Hexagonal pattern obtained in a cylindrical cell with aspect ratio $\Gamma=60$. The values of the parameters used are $g_{2}=0.35$ and $\epsilon=0.3$. A non-zero forcing localized at the boundary has been used. For weak forcing (a), $f=0.1$, two domains of hexagonal symmetry with different orientation appear that meet at a grain boundary. For strong forcing (b), $f=0.5$, a uniform hexagonal pattern is obtained at long times.

Figure 3. We observe a transition from hexagons to rolls by changing $\epsilon$ from $\epsilon=0.1$ to $\epsilon=0.3$, in a cylindrical cell with an aspect ratio $\Gamma=60$. The initial condition in a uniform hexagonal pattern like the one shown in Fig. 2b. Two different times, $t=1712(\mathrm{a})$, and $t=1819$ (b) are shown. The rolls appearing near the defects and grain boundaries propagate along the axes which adjoin the defects.

Figure 4. Stationary spiral obtained in a cylindrical cell with aspect ratio $\Gamma=60$, with $g_{2}=0.35, \epsilon=0.2$ and $f=0.1$. Concentric rolls are deformed by the nucleation of defects which ultimately lead to a spiral pattern. 